\title{
Clinical and multimodal biomarker correlates of ADNI neuropathological findings
}

\author{
Jon B Toledo ${ }^{1 \dagger}$, Nigel J Cairns ${ }^{2 \dagger}$, Xiao Da ${ }^{3}$, Kewei Chen ${ }^{4}$, Deborah Carter ${ }^{2}$, Adam Fleisher ${ }^{4}$, Erin Householder ${ }^{2}$, \\ Napatkamon Ayutyanont ${ }^{4}$, Auttawut Roontiva ${ }^{4}$, Robert J Bauer ${ }^{4}$, Paul Eisen ${ }^{5}$, Leslie M Shaw ${ }^{1}$, Christos Davatzikos ${ }^{3}$, \\ Michael W Weiner ${ }^{6}$, Eric M Reiman ${ }^{4}$, John C Morris ${ }^{2 *}$, John Q Trojanowski ${ }^{1 *}$ and for the Alzheimer's Disease \\ Neuroimaging Initiative
}

\begin{abstract}
Background: Autopsy series commonly report a high percentage of coincident pathologies in demented patients, including patients with a clinical diagnosis of dementia of the Alzheimer type (DAT). However many clinical and biomarker studies report cases with a single neurodegenerative disease. We examined multimodal biomarker correlates of the consecutive series of the first 22 Alzheimer's Disease Neuroimaging Initiative autopsies. Clinical data, neuropsychological measures, cerebrospinal fluid A 3 , total and phosphorylated tau and a-synuclein and MRI and FDG-PET scans.

Results: Clinical diagnosis was either probable DAT or Alzheimer's disease (AD)-type mild cognitive impairment (MCI) at last evaluation prior to death. All patients had a pathological diagnosis of AD, but only four had pure AD. A coincident pathological diagnosis of dementia with Lewy bodies (DLB), medial temporal lobe pathology (TDP-43 proteinopathy, argyrophilic grain disease and hippocampal sclerosis), referred to collectively here as MTL, and vascular pathology were present in $45.5 \%, 40.0 \%$ and $22.7 \%$ of these patients, respectively. Hallucinations were a strong predictor of coincident DLB (100\% specificity) and a more severe dysexecutive profile was also a useful predictor of coincident DLB (80.0\% sensitivity and $83.3 \%$ specificity). Occipital FDG-PET hypometabolism accurately classified coincident DLB (80\% sensitivity and 100\% specificity). Subjects with coincident MTL showed lower hippocampal volume.
\end{abstract}

Conclusions: Biomarkers can be used to independently predict coincident AD and DLB pathology, a common finding in amnestic MCl and DAT patients. Cohorts with comprehensive neuropathological assessments and multimodal biomarkers are needed to characterize independent predictors for the different neuropathological substrates of cognitive impairment.

Keywords: Alzheimer's disease, Mild cognitive impairment, CSF, MRI, Autopsy, Neuropathology, Dementia, Biomarkers, Amyloid, Tau

\section{Background}

Studies based on clinical and neuropathological diagnoses have shown that Alzheimer's disease (AD) is the most common cause of dementia [1-4]. However, there are several neurodegenerative [5-8] and non-neurodegenerative pathologies [1,9-12] that are known to contribute to cognitive impairment and a dementia diagnosis. Different

\footnotetext{
*Correspondence: morrisj@abraxas.wustl.edu; trojanow@mail.med.upenn.edu ${ }^{\dagger}$ Equal contributors

2Departments of Neurology and Pathology \& Immunology, Washington University School of Medicine, St. Louis, MO, USA

'Department of Pathology \& Laboratory Medicine, Institute on Aging, Center for Neurodegenerative Disease Research, University of Pennsylvania Perelman School of Medicine, Philadelphia, PA, USA

Full list of author information is available at the end of the article
}

clinical dementia syndromes have degrees of clinicopathological correlation, therefore if clinical diagnosis is used to estimate the accuracy of biomarkers and their cutoffs, inaccurate results may occur [13-15]. In addition, coincident neurodegenerative diseases (NDDs) and vascular pathology are common findings in subjects with AD in autopsy series [1,9,14,16-18]. However, clinical studies of dementia of the Alzheimer type (DAT) and other NDDs assign a single primary clinical diagnosis to patients. Accordingly, most biomarker studies are based on clinical diagnoses and report results on subjects using a single NDD diagnosis as the outcome. While the use of clinical diagnoses is helpful for the screening and the evaluation of new biomarkers, substantial follow up studies are 
needed to establish the performance of these biomarkers. Such studies should include in the analysis consecutive series of patients with NDDs and non-NDDs together with multimodal biomarkers assessing their performance in complex settings with several coincident diseases. Previously, retrospective studies have analyzed the correlation between neuropathological findings and CSF $[14,19,20]$, magnetic resonance imaging (MRI) [21-24] and positron emission tomography (PET) [21,25-27]. Most of these studies tried to categorize patients into a single diagnostic category. Here, we instead tried to assess how different combinations of biomarkers can detect different coincident pathologies and therefore predict the different combinations of neuropathological substrates of the cognitive impairment in the studied subjects to help identify homogeneous cohorts of patients for clinical studies and clinical trials. This is especially true of clinical trials for DAT in which one pathology is targeted for study such as therapies that target $A \beta$ or tau mediated mechanisms of neurodegeneration. Thus, here we specifically tested cerebrospinal fluid (CSF) biomarkers for the diagnosis of $\mathrm{AD}$ $\left(\mathrm{A} \beta_{1-42}\right.$, total tau (t-tau) and phosphorylated tau ( $\left.\mathrm{p}-\mathrm{tau}_{181}\right)$ and dementia with Lewy bodies (DLB) ( $\alpha$-synuclein), MRI hippocampal and occipital pathology for the diagnosis of coincident DLB and medial temporal lobe (MTL) pathologies and occipital hypometabolism for the diagnosis of DLB. In addition, we tested the neuropathological association of hallucinations, memory and executive dysfunction. For this study, we examined the first 22 patients in the Alzheimer's Disease Neuroimaging Initiative (ADNI) who were longitudinally followed to death and underwent postmortem examination.

\section{Methods}

\section{Participants and neuropsychological testing}

Data used in the preparation of this article, was downloaded from the ADNI database August 19th 2013. The ADNI was launched in 2004 by the National Institute on Aging (NIA), the National Institute of Biomedical Imaging and Bioengineering (NIBIB), the Food and Drug Administration, private pharmaceutical companies and non-profit organizations as reviewed elsewhere [28] (see additional information in http://www.adni-info.org and Additional file 1: Table S1). Here, we focused on the first 22 ADNI subjects who came to autopsy, i.e. 21 from ADNI 1 and one subject from ADNI 2 (Table 1 and Additional file 1: Table S3). A diagnosis of MCI was established as previously described [29,30] and DAT was based on the National Institute of Neurological and Communicative Disorders and Stroke-Alzheimer's Disease and Related Disorders Association criteria for probable AD [29,31]. Summary composite executive and memory measures developed by Gibbons et al. [32] and Crane et al. [33], respectively, were used to estimate the cognitive profile differences associated with the neuropathological diagnoses.

\section{CSF biomarker collection and analysis}

Baseline CSF samples were obtained using polypropylene collection and transfer tubes in the morning after an overnight fast and processed as described (Additional file 1) $[34,35] . A \beta_{1-42}, t$-tau, and $\mathrm{p}-$ tau $_{181}$ were measured using the multiplex xMAP Luminex platform (Luminex Corp, Austin, TX) with Innogenetics (INNO-BIA AlzBio3; Ghent, Belgium; for research use-only reagents) immunoassay kit-based reagents. The capture monoclonal antibodies used were 4D7A3 for A $\beta_{1-42}$, AT120 for t-tau and AT270 for $\mathrm{p}$-tau $\mathrm{t}_{181}$. The analyte-specific detector antibodies were HT7, for tau, and 3D6, for the N-terminus of A $\beta$ (immunoassay performance details described in Shaw et al. [35] and Additional file 1). For the $\alpha$-syn assay, Luminex MicroPlex Microspheres (Luminex Corp, Austin, TX) were chemically coated with rabbit anti- $\alpha$-syn antibody ASY-1 and biotinylated goat anti-human $\alpha$-syn antibody (R\&D systems, Minneapolis, MN, USA) was used as the detection antibody [36,37].

\section{Magnetic resonance imaging acquisition and processing}

Acquisition of 1.5-T MCI data at each performance site for the 21 ADNI 1 subjects followed a previously described standardized protocol that included a sagittal volumetric 3D MPRAGE with variable resolution around the target of $1.2 \mathrm{~mm}$ isotropically. The scans subjected to several correction methods including gradwarp, B1 calibration, N3 correction, and (in-house) skull-stripping (see http://adni.loni.usc.edu/ and [38] for further details). The images were processed with a freely-available pipeline [39] (for software, see www.rad.upenn.edu/sbia). Briefly, images were segmented into 3 tissue types: GM white matter (WM), and CSF. After a high-dimensional image warping to an atlas, regional volumetric maps for GM, WM and CSF were created, referred to herein as RAVENS maps. RAVENS maps are used for voxel-based analysis and group comparisons of regional tissue atrophy, as well as for constructing an index of $\mathrm{AD}$ brain morphology.

\section{FDG-PET acquisition}

FDG-PET data was acquired and reconstructed with the use of measured-attenuation correction and the specified reconstruction algorithm for each scanner type according to a standardized protocol (http://adni.loni.usc.edu/datasamples/access-data/). All images were pre-processed by ADNI PET Coordinating Center investigators at the University of Michigan and uploaded to the LONI ADNI website. These images were downloaded by investigators at Banner Alzheimer's Institute for additional pre-processing using SPM5 (http://www.fil.ion.ucl.ac.uk/ spm) for computation of the HCI, for voxel-wise group comparison and by the investigators at U. Penn for the primary FDG-PET measure of left/right occipital regions. 
Table 1 Subject characteristics

\begin{tabular}{|c|c|c|c|c|c|}
\hline & $A D$ & $\begin{array}{l}A D+M T L \\
\text { pathology }\end{array}$ & $A D+D L B$ & $\begin{array}{l}A D+D L B+M T L \\
\text { pathology }\end{array}$ & p-value \\
\hline & $n=(7)$ & $n=(5)$ & $n=(6)$ & $n=(4)$ & \\
\hline \multirow{4}{*}{$\begin{array}{l}\text { Longitudinal clinical } \\
\text { diagnosis }\end{array}$} & $1 \mathrm{CN}$ to $\mathrm{MCl}$ & $1 \mathrm{MCl}$ stable & $1 \mathrm{MCl}$ to DAT & $2 \mathrm{MCl}$ to DAT & - \\
\hline & $2 \mathrm{MCl}$ stable & $3 \mathrm{MCl}$ to DAT & & & \\
\hline & $2 \mathrm{MCI}$ to DAT & $1 \mathrm{DAT}$ & 5 DAT & 2 DAT & \\
\hline & 2 DAT & & & & \\
\hline \multirow{8}{*}{$\begin{array}{l}\text { Neuropathological } \\
\text { diagnosis }\end{array}$} & $1 \mathrm{LNC} A \mathrm{AD}$ & $1 \mathrm{HNC}$ AD + MTL-TDP & $1 \mathrm{HNC} A D+\mathrm{DLB}$ & 1 DLB-LNC & - \\
\hline & $3 \mathrm{HNC}$ AD & & & $A D+A G D$ & \\
\hline & 3 HNC AD + SVD-I & $\begin{array}{l}2 \text { LNC AD + AGD } \\
+ \text { MTL-TDP }\end{array}$ & $3 \mathrm{HNC}$ & $\begin{array}{l}1 \text { DLB- LNC AD } \\
+ \text { MTL-TDP }\end{array}$ & \\
\hline & & $\begin{array}{l}1 \mathrm{HNC} A \mathrm{AD}+\mathrm{AGD} \\
+\mathrm{MTL}-\mathrm{TDP}+\mathrm{HS}\end{array}$ & & $\begin{array}{l}2 \text { HNC AD + DLB } \\
+ \text { MTL-TDP }\end{array}$ & \\
\hline & & $\begin{array}{l}1 \mathrm{HNC} \text { AD + AGD } \\
+\mathrm{MTL}-\mathrm{TDP}+\mathrm{HS}+\mathrm{SVD}-\mathrm{I}\end{array}$ & & & \\
\hline & & & $A D-+D L B$ & & \\
\hline & & & 1 HNC AD-DLB + SVD-I & & \\
\hline & & & $1 \mathrm{DLB}+\mathrm{LNC} A \mathrm{AD}$ & & \\
\hline Age at death (years) ${ }^{2}$ & $80(77-83)$ & $86(82-88)$ & $80.5(72.25-83.75)$ & $81(77.75-84.75)$ & 0.41 \\
\hline Gender (n male/total) & $3 / 7$ & $4 / 5$ & $6 / 6$ & $4 / 4$ & 1.0 \\
\hline Education (years) $^{1}$ & $15.4(2.4)$ & $15.0(2.4)$ & $16.1(2.6)$ & $14.0(2.3)$ & 0.62 \\
\hline $\begin{array}{l}\text { Baseline visit to death } \\
\text { (weeks) }^{2}\end{array}$ & $240.7(98.7-256.8)$ & $181.9(108.4-289.0)$ & $136.5(80.9-278.3)$ & $234.9(189.1-257.1)$ & 0.72 \\
\hline APOE ع4 (n positive/total) & $4 / 7$ & $1 / 5$ & $5 / 6$ & $1 / 3$ & 0.18 \\
\hline $\begin{array}{l}\text { ADAS-Cog (13 item) } \\
\text { baseline }\end{array}$ & $20.0(10.3-28.0)$ & $22.0(17.7-29.0)$ & $33.3(18.0-54.7)$ & $30.3(25.3-35.0)$ & 0.041 \\
\hline$A \beta_{1-42}(\mathrm{pg} / \mathrm{mL})^{2}$ & $134.0(86.0-261.0)$ & $249.0(123.0-261.0)$ & $138.0(82.0-152.0)$ & $171.0(134.0-201.9)$ & 0.59 \\
\hline T-Tau $(p g / m L)^{2}$ & $141.5(60.0-274.0)$ & $65.0(55.0-89.0)$ & $88.0(37.0-154.0)$ & $73.0(56.0-103.6)$ & 0.61 \\
\hline P-Tau $181(\mathrm{pg} / \mathrm{mL})^{2}$ & $51.0(17.0-70.0)$ & $22.0(12.0-33.0)$ & $28.0(11.0-45.0)$ & $21.0(19.0-24.0)$ & 0.32 \\
\hline
\end{tabular}

${ }^{1}$ Mean (Standard Deviation); ${ }^{2}$ Median (Minimum-Maximum).

AGD: Argyrophilic grain disease; SVD-I: Small vessel disease (arteriolosclerosis and/or cerebral amyloid angiopathy) and one or more infarcts); HNC AD: High neuropathologic change Alzheimer's disease; HS: Hippocampal sclerosis; LNC AD: Low neuropathological change Alzheimer's disease; MTL-TDP: TDP pathology circumscribed to the medial temporal lobe.

HCI is a summary measure that reflects how the pattern and magnitude of cerebral hypometabolism in an individual's FDG-PET image corresponds to that in probable AD patients. It was computed as previously described [40]. In addition to this global single index and as our primary FDG-PET measure, the relative cerebral metabolic rate for glucose (CMRgl) from anatomically predefined left and right occipital regions of interest (ROIs) was also extracted with global counts as reference region. Group comparisons were carried out also using a voxelwise two-sample independent t-test implemented in SPM5 accounting for the whole brain PET counts variations using proportional scaling.

\section{Neuropathological procedures and diagnosis}

The ADNI Neuropathology Core (ADNI-NPC) was funded and established at Washington University in St. Louis in September 2007; therefore no autopsies were performed on deceased ADNI subjects prior to this time. Established procedures for obtaining informed consent for autopsy and the autopsy procedures has been previously described [41]. Briefly, participating centers undertake their own brain assessment and provide standard sets of fixed tissue blocks/sections and frozen sections to the ADNINPC or send the brain to the ADNI-NPC if the center does not routinely perform neuropathological assessments. Formalin-fixed paraffin embedded tissue blocks are obtained from the left cerebrum for the following 16 areas: middle frontal gyrus, superior and middle temporal gyri, inferior parietal lobe (angular gyrus), occipital lobe to include the calcarine sulcus and peristriate cortex, anterior cingulate gyrus at the level of the genu of the corpus callosum, posterior cingulate gyrus and precuneus at the level of the splenium, amygdala and entorhinal cortex, hippocampus and parahippocampal gyrus at the level of the lateral geniculate nucleus, striatum (caudate 
nucleus and putamen) at the level of the anterior commissure, lentiform nucleus (globus pallidus and putamen), thalamus and subthalamic nucleus, midbrain, pons, medulla oblongata, cerebellum with dentate nucleus and spinal cord when available.

Sections from the different blocks were stained using hematoxylin and eosin and a modified Bielschowsky silver impregnation; immunohistochemistry (IHC) was performed using antibodies to ubiquitin (Dako, Carpinteria, CA), phosphorylated tau (PHF1, a gift from Dr. P. Davies, Albert Einstein College of Medicine, Yeshiva University, $\mathrm{NY}$ ), A $\beta$ (10D5, Eli Lilley, Indianapolis, IN), phosphorylated $\alpha$-synuclein (Wako, Richmond, VA), and phosphorylated TDP-43 (Cosmo Bio, Carlsbad, CA) using methods previously described [41]. The neuropathological diagnosis for each case was determined in accordance with previously described criteria [7,42] for the pathological diagnosis of $\mathrm{AD}$ using the diagnostic nomenclature of the National Alzheimer's Coordinating Center for diagnostic neuropathology [43] (DAT refers to the clinical diagnosis, whereas $\mathrm{AD}$ refers to the neuropathological diagnosis in this manuscript). Notably, this allows for a single primary neuropathological diagnosis and any other coincident secondary neuropathological diagnoses in addition to the primary neuropathological diagnosis [43]. In addition, Braak neurofibrillary tangle (NFT) staging [44], Consortium to Establish a Registry for AD (CERAD) scores [45], semiquantitative ratings for neuritic and diffuse plaques, Lewy body disease stage and the probability of DLB [46] were recorded. The Cairns et al. [47] and Mackenzie et al. [6] criteria and the National Institute on Aging-Alzheimer's Association guideline [48] were applied for the diagnosis of frontotemporal lobar degeneration (FTLD) and AD, respectively. Due to the small number of cases and the heterogeneity of diagnoses, we grouped HS, AGD and MTL-TDP deposition under the category MTL pathologies. In addition, five cases had a pathological diagnosis of SVD-I.

\section{Statistical analysis}

For the comparison of the four diagnostic groups listed in Table 1 Kruskall-Wallis test was applied to nonnormally distributed variables and ANOVA was applied for those with a normal distribution. For further analyses, a Box-Cox transformation was applied to nonnormally distributed variables and parametric tests were applied. To analyze association between two quantitative variables, Pearson correlation coefficient was used. Braak stages were dichotomized into a low (I/II) and high (V/ VI) group. A logistic regression model was applied for multivariable analyses in case of a binary dependent variable (DLB positivity, e.g.) or a linear regression in case of quantitative dependent variables. Cutoffs for classification models were selected to maximized sensitivity and specificity. All statistical tests were two-sided. Most of our analyses were specified a priori based on previous findings (occipital hypometabolism in DLB and differences in hippocampal volume) and were not corrected for multiple comparisons. For multiple comparison adjustment of tests not based on an a priori hypothesis, the Holmes correction was used for non-imaging (i.e. for the cognitive tests) or non voxel-based imaging data. Statistical significance was set at the $p<0.05$ level. For secondary voxel-wise analysis, uncorrected $\mathrm{p}=0.005$ was used.

\section{Results}

\section{Brain donation status in ADNI and ADNI autopsy rates}

Information about the participation in the brain donation program is available on a total of 1119 ADNI subjects (who were recruited in ADNI-1, ADNI-GO and ADNI-2): 653 subjects have made a decision to donate their brains at death, 5 are reviewing the information and 461 have not made a decision. Of the 653 who have made a decision, 139 (21.1\%) are not participating in the autopsy program. The main reason not to take part in this brain donation program was the unwillingness of the subjects in 59 cases, followed by the lack of logistics in the study site in 31 cases, the participation of the patient in another autopsy program in 15 cases, the inconvenience and burden for the family in 9 cases and religious reasons in another 9 cases. Of the 514 cases who agreed to participate, 327 signed the provisional consent, 61 are reviewing the form and for 126 subjects the form will be completed at time of autopsy. Finally, 285 of the 461 subjects who have not made a decision have been given information about the program and 176 have not been approached. Up to the data download time on 17 August, 2013 there were 61 known deceased ADNI subjects, although deaths that occurred in subjects lost to follow-up is not always known. We compared the ADNI 1 subjects who died and came to autopsy to those who died with an autopsy and those that remain alive (or were lost to follow-up and no information about death is available). Dead patients who died without autopsy or remained alive were less cognitively impaired that those that came to autopsy (Additional file 1: Table S2).

\section{Neuropathological findings}

The primary neuropathological diagnosis was $\mathrm{AD}$ for 19 cases and DLB for 3; all 22 cases met diagnostic criteria for $\mathrm{AD}$, including 12 who were clinically diagnosed with mild cognitive impairment (MCI) due to AD (Table 1). However, only 4 cases had a single AD neuropathological diagnosis, whereas all other cases had two or more neuropathological diagnoses, with $\mathrm{AD}$ and DLB being the most common comorbidities (10 cases). Other coincident diagnoses included: small vessel disease with infarcts (SVD-I), argyrophilic grain disease (AGD), 
MTL-TDP and hippocampal sclerosis (HS). In addition, 5 cases had coincident SVD-I. The TDP pathology was confined to amygdala, entorhinal cortex and dentate gyrus in all $\mathrm{AD}+\mathrm{DLB}+\mathrm{MTL}-\mathrm{TDP}$ cases. In the AD + MTL pathology group TDP pathology was confined to amygdala, entorhinal cortex and dentate gyrus in two cases, one case had a low degree of TDP pathology in CA 1 region and the last case had a low degree of TDP pathology in CA1 and neocortical regions. For the purpose of this study subjects were grouped into AD and AD + DLB (irrespective of which one was the primary diagnosis). These groups were further stratified based on the absence or presence of additional comorbidities affecting the MTL as summarized in Table 1. All cases except one AD + DLB case (without any cerebral amyloid angiopathy) had mild or moderate cerebral amyloid angiopathy. Four cases in the AD group had severe atherosclerosis whereas other cases had none or mild atherosclerosis. A detailed neuropathological description of all 22 cases can be found in Additional file 1: Table S3 and 'heatmaps' that summarize the neuropathological findings in the different groups are displayed in Figure 1. The only clinical difference between groups was a greater cognitive impairment as measured by the ADAS-Cog in the AD + DLB and AD + DLB + MTL-TDP groups compared to the $\mathrm{AD}$ and $\mathrm{AD}+\mathrm{MTL}-\mathrm{TDP}$ groups (Table 1 ).

\section{Clinical findings}

At baseline, there were one cognitively normal subject $(\mathrm{CN})$, eleven MCI and ten DAT patients. At the last visit prior to death, the $\mathrm{CN}$ subject had converted to $\mathrm{MCI}$ and eight MCI subjects had converted to DAT. All 18 subjects who died with a dementia diagnosis were diagnosed as DAT probable and the 3 stable $\mathrm{MCI}$ and the single CN subject who converted to MCI were diagnosed as MCI due to DAT. One amnestic MCI patient was noted to have depression which required drug treatment and three patients had parkinsonian signs (their neuropathological diagnoses were $\mathrm{AD}+\mathrm{DLB}, \mathrm{AD}+$ DLB + MTL-TDP and AD + MTL-TDP). Finally, the patient with AD + SVD-I + AGD + MTL-TDP + HS had significant behavioral impairments.

Whereas most of the subjects in the four groups were demented in their last visits before death, the degree of impairment varied between the groups at the baseline visit. Therefore, we compared neuropsychological performance at the last neuropsychological evaluation before death (median time between last visit and time of death 49.6 weeks, interquartile range 30.1-102.7 weeks) (Table 2). Higher Braak stages were associated with worse memory sum score $\left(\mathrm{t}=-3.04, \mathrm{p}_{\text {adjusted }}=0.026\right)$ (Figure 2a) and coincident DLB $(\mathrm{t}=-3.06$, padjusted $=0.024)$ was associated with worse executive function (Figure $2 \mathrm{~b}$ ). No associations were found for SVD-I and/or MTL-TDP. Finally, we developed a mismatch score subtracting the executive summary score from the memory summary score. Therefore, subjects with a positive score would show predominant dysexecutive profile, whereas subjects with negative values have more severe memory impairment. The presence of coincident DLB was associated with a higher score $(\mathrm{t}=3.7, \mathrm{p}=0.0015)$ and a mismatch cutoff score of 0.38 was associated with a sensitivity of $80.0 \%$ and a specificity of $83.3 \%$ to detect the presence of coincident DLB pathology (Figure 2c). When we analyzed the baseline visit the difference using the same cutoff and the cutoff retained its specificity but the sensitivity dropped (Sensitivity $70.0 \%$ and Specificity $83.3 \%$ ) (Figure 2d).

There were no differences in the Neuropsychiatric Inventory Questionnaire (NPI-Q) total score based on the different neuropathological characteristics (Figure 2e, Table 2). Conversely, there was a strong association between the presence of hallucinations (item of the NPIQ-Q) and a coincident DLB ( $\mathrm{p}=0.0002)$; all four subjects who presented hallucinations in their last visit had a DLB (Sensitivity of $40 \%$ and specificity of $100 \%)$.

\section{CSF Biomarker findings}

CSF $A \beta_{1-42}$, t-tau and $\mathrm{p}$-tau $\mathrm{t}_{181}$ measurements were available for 15 of the 22 deceased ADNI patients (Additional file 1: Table S3). As seen in Figure 3a all subjects had NIA-AA criteria A score of 2 or 3 using the recent NIA-AA criteria (For one subject Thal phase could not be estimated) [48]. Three subjects had CSF A $\beta_{1-42}$ values equal or above $240 \mathrm{pg} / \mathrm{mL}$ (two of these subjects had a Braak stage I and the last one had a Braak stage II), whereas the remaining patients had values below $202 \mathrm{pg} / \mathrm{mL}$. Both $\mathrm{t}$-tau $(\mathrm{r}=0.59, \mathrm{p}=0.0195)$ (Figure $3 \mathrm{~b}$ ) and $\mathrm{p}-\operatorname{tau}_{181}(\mathrm{r}=0.70, \mathrm{p}=0.0035)$ (Figure $3 \mathrm{c}$ ) correlated with Braak stage. Neither the presence of coincident DLB nor the presence of coincident MTL pathologies showed an association with CSF biomarker levels. 13 subjects had CSF $\alpha$-synuclein measurements, but no differences were found for the $\alpha$-synuclein $\mathrm{p}$-tau181 mismatch $(\mathrm{t}=-1.31, \mathrm{p}=0.22)$ (Figure $3 \mathrm{~d}$ ) and $\alpha$-synuclein $(\mathrm{t}=-1.59, \mathrm{p}=0.16)$ (Figure 3e) based on the presence or absence of DLB.

\section{Neuroimaging findings}

Due to the small number of autopsied patients with amyloid neuroimaging PET scans, neither Pittsburgh B compound (PiB) PET nor AV45 PET scans were analyzed. On the other hand, 11 patients (Additional file 1: Table S3), 6 without DLB and 5 with comorbid DLB, had FDG-PET occipital lobe measures and 15 subjects had HCI measures available from FDG-PET performed at the baseline visit. 
Table 2 Association between neuropathological findings and neuropsychological and neuropsychiatric measures

\begin{tabular}{lllll}
\hline & Braak stage (I/II vs. V/VI) & Comorbid DLB & Comorbid MTL pathologies & Comorbid SVD-I \\
\hline Memory sum score & $t=-3.01$ & $t=0.29$ & $t=-1.64$ & $t=0.80$ \\
& $p=0.0070$ & $p=0.7795$ & $p=0.1198$ & $p=0.449$ \\
Executive function sum score & $r=-0.34$ & $t=2.70$ & $t=-0.76$ & $t=1.15$ \\
& $p=0.1326$ & $p=0.01423$ & $p=0.4553$ & $p=0.2922$ \\
NPI-Q & $r=-0.33$ & $t=0.65$ & $t=0.70$ & $p=-0.76$ \\
& $p=0.1632$ & $p=0.5239$ & $p=0.4928$ & $p=0.4553$ \\
\hline
\end{tabular}

P-values are adjusted for multiple comparisons.

Consistent with previous reports [21,25,27], DLB patients showed a significant right $(\mathrm{t}=-3.27, \mathrm{p}=0.0097)$ (Figure $4 \mathrm{a}$ ) and left $(\mathrm{t}=-4.47, \mathrm{p}=0.0016)$ (Figure $4 \mathrm{~b})$ occipital lobe hypometabolism. After adjusting for ADAS-Cog score only the left occipital hypometabolism was significantly lower in the cases with DLB (right occipital metabolism: $\mathrm{t}=-2.21, \mathrm{p}=0.058$; left occipital metabolism: $\mathrm{t}=-2.32$, $\mathrm{p}=0.041$ ) and differences also remained significant after adjusting for time to death (right occipital metabolism: $\mathrm{t}=-3.78, \mathrm{p}=0.0054$; left occipital metabolism: $\mathrm{t}=-4.67$, $\mathrm{p}=0.0016)$. The same cutoff value of 1.48 showed a sensitivity of $80 \%$ and a specificity of $100 \%$ to classify patients with DLB based on either the left or right occipital regions. Interestingly the AD-DLB case that was classified as $\mathrm{AD}$ had the lowest burden of LBs that were circumscribed to the amygdala, entorhinal cortex and midbrain, whereas all the other cases were diffuse neocortical cases. In addition, the presence of DLB $(t=2.54, p=0.026)$, but not

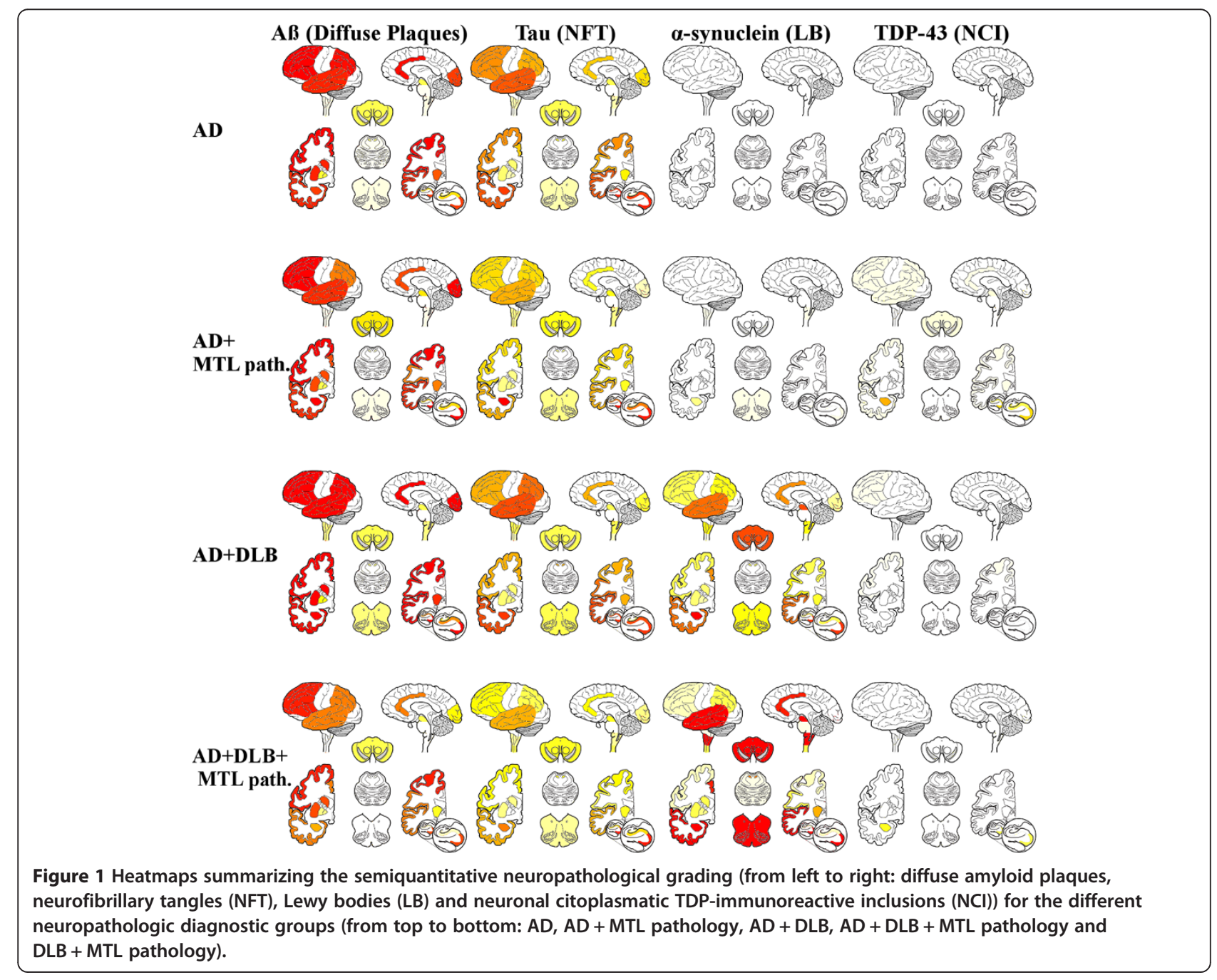




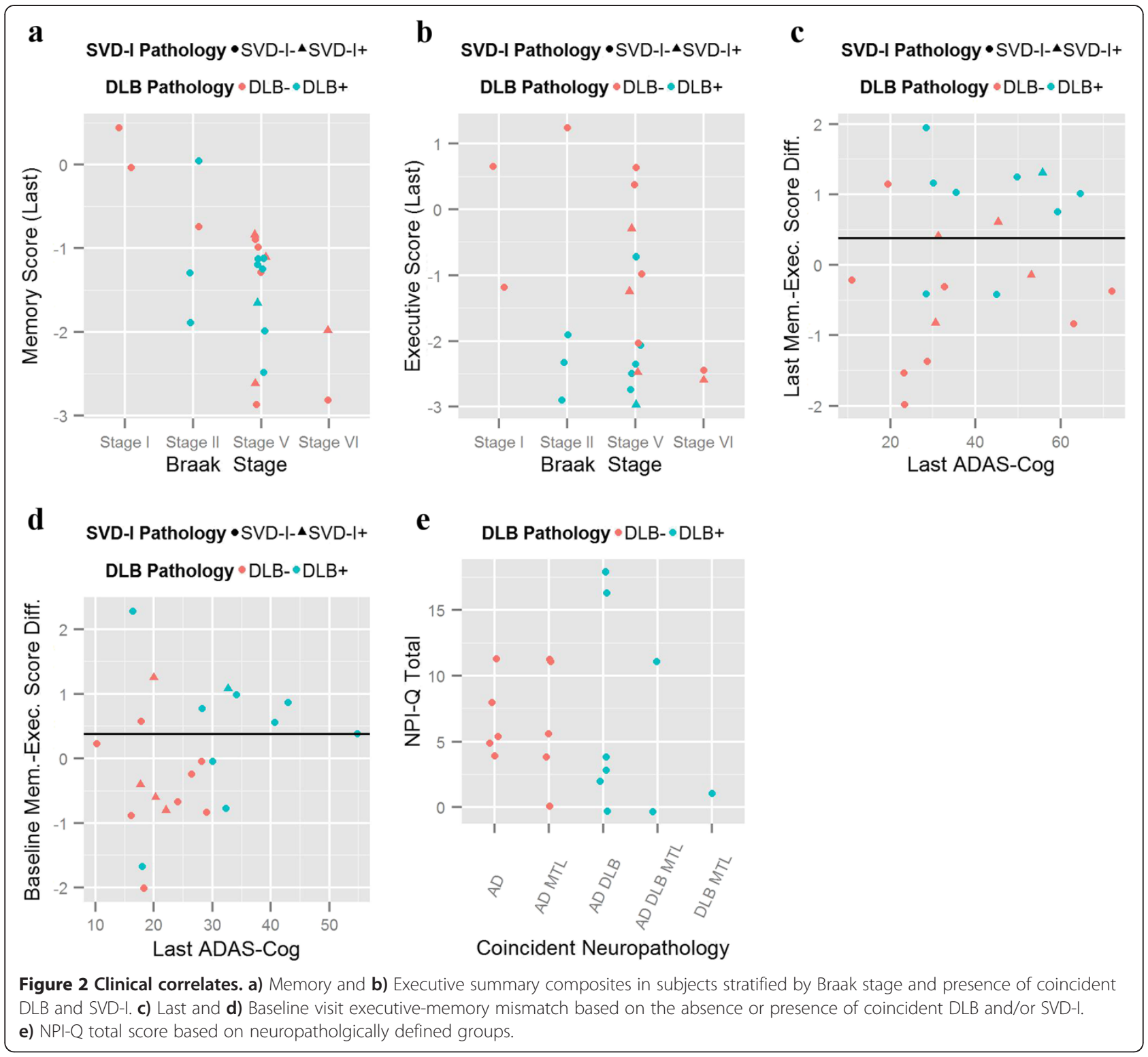

higher Braak stage $(\mathrm{t}=0.78, \mathrm{p}=0.44)$, was associated with higher hypometabolic convergence index (HCI) values (Figure 4c). This association remained significant when the presence of a DLB diagnosis was further adjusted for Braak stage $(t=2.47, p=0.031)$, the ADAS-Cog score at the time of $\operatorname{scan}(\mathrm{t}=2.32, \mathrm{p}=0.041)$ or time to death $(t=2.71, p=0.020)$. Finally, we conducted a secondary voxel-wise group comparison covarying out ADAS-Cog score for disease severity and found several hypometabolic areas with coincident DLB as shown in Figure 5 and listed in the Additional file 1: Table S5

We studied two MRI areas selected a priori using the MRI gray matter (GM) volume adjusting for total intracranial volume (ICV): the occipital lobe, based on previous fluorodeoxyglucose (FDG)-PET findings, and the hippocampal volume, based on previous MRI findings. Neither left $(t=1.2, p=0.24)$ or right $(t=1.48, p=0.16)$ hippocampal volume, nor left $(t=-0.09, \mathrm{p}=0.93)$ or right $(t=0.003, p=1.0)$ occipital GM volume showed differences in subjects with coincident DLB in the baseline MRI (Figure 4d-g). Finally, we repeated the analysis of the MRI occipital and occipital GM volumes using different visits per subject based on a matched ADAS-Cog score (Additional file 1: Figure S1) and again found no group differences (Additional file 1: Table S4). In addition, there no correlation was found between the occipital GM volume and the occipital FDG CMRgl (left occipital lobe: $\mathrm{r}=0.27, \mathrm{p}=0.42$; right occipital lobe: $\mathrm{r}=0.23, \mathrm{p}=$ 0.50) (Figure $4 \mathrm{~h}$ ).

Finally, we tested the association of MTL-TDP on hippocampal volume. Because the neuropathologically 

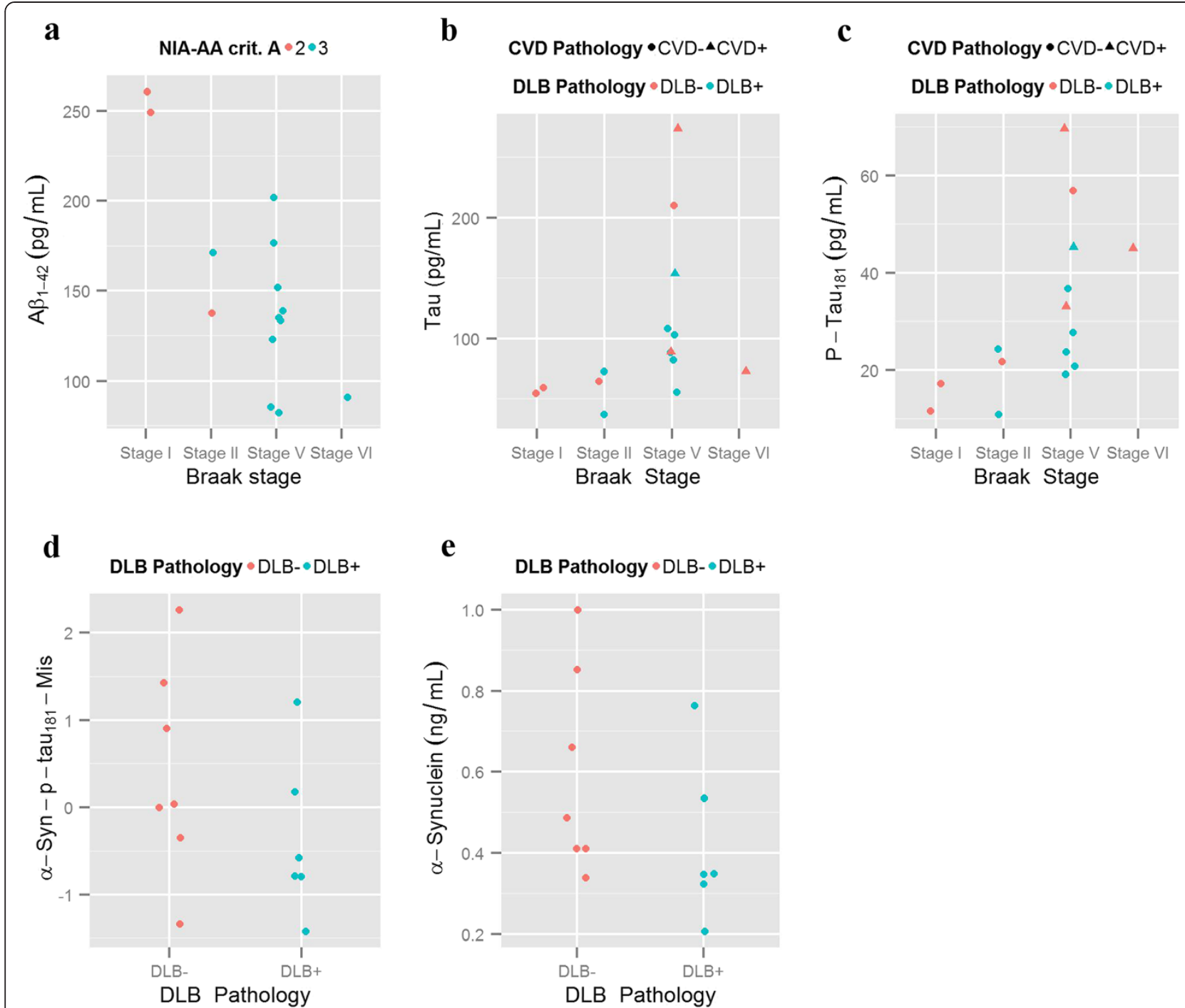

Figure 3 CSF biomarker correlates. a) $A \beta_{1-42}$ levels based on a) NIA-AA criteria A score and Braak score. b)T-tau and c) P-tau ${ }_{181}$ levels based on Braak stage. d) a-Synuclein-p-tau 181 mismatch and e) a-Synuclein based on presence or absence of coincident DLB and SVD-I.

studied hemisphere was the left one, we only examined the association with the left hippocampal volume using MRI scan acquired at the times with matched clinical severity. In the analysis that include Braak stage and presence of MTL-TDP there was a significant association between left hippocampal atrophy and MTL-TDP $(t=-2.75$, $\mathrm{p}=0.013)$ and a trend with Braak stage $(\mathrm{t}=-1.86, \mathrm{p}=$ 0.080) (Figure 4i).

\section{Prediction of DLB pathology in the ADNI cohort}

A total of 76 DAT patients and 139 MCI ADNI-1 subjects had occipital lobe hypometabolism measures and 39.5\% and $11.5 \%$ of them had occipital hypometabolism below the 1.48 cutoff, respectively. Of the 193 ADNI-1 DAT subjects a total of $30.1 \%$ had a predominantly executive cognitive impairment which would be characteristic of non-DAT pathology.

\section{Discussion}

We performed a multimodal biomarker analysis of consecutive ADNI subjects who came to autopsy after longitudinal follow-up to death. Most of the subjects were late amnestic MCI and probable DAT at baseline visit, without any atypical clinical presentation. In addition, subjects with vascular disease or a Hachinski Ischemic Score $>4$ were excluded from the ADNI study [29]. Despite this, only four out of 22 subjects had a neuropathological diagnosis of pure AD (13.6\%), while DLB, MTL-TDP and infarcts were present in 45.5\%, 40.9\% and $22.7 \%$ of the patients, respectively. A predominantly executive dysfunction was associated with the presence 


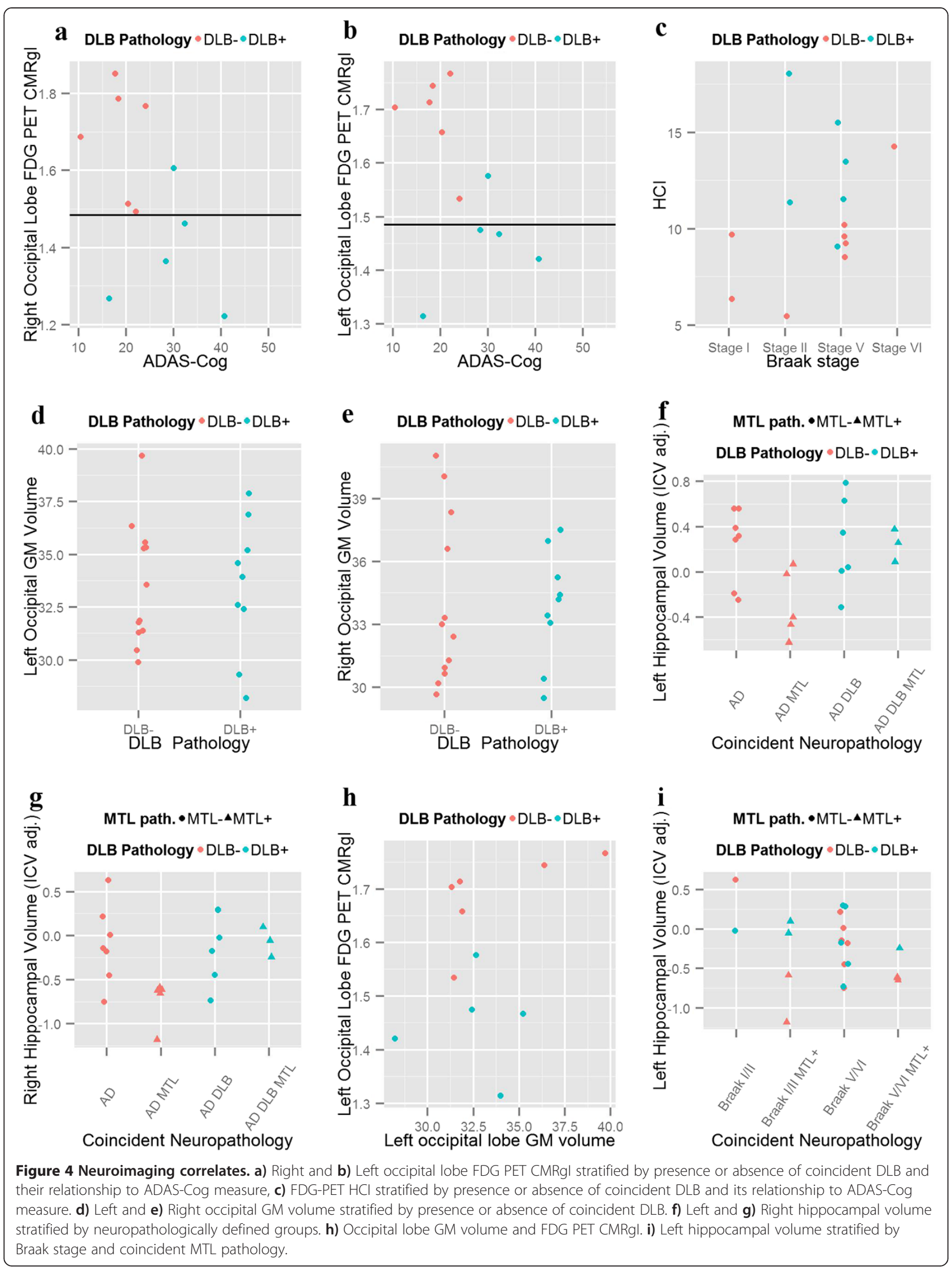




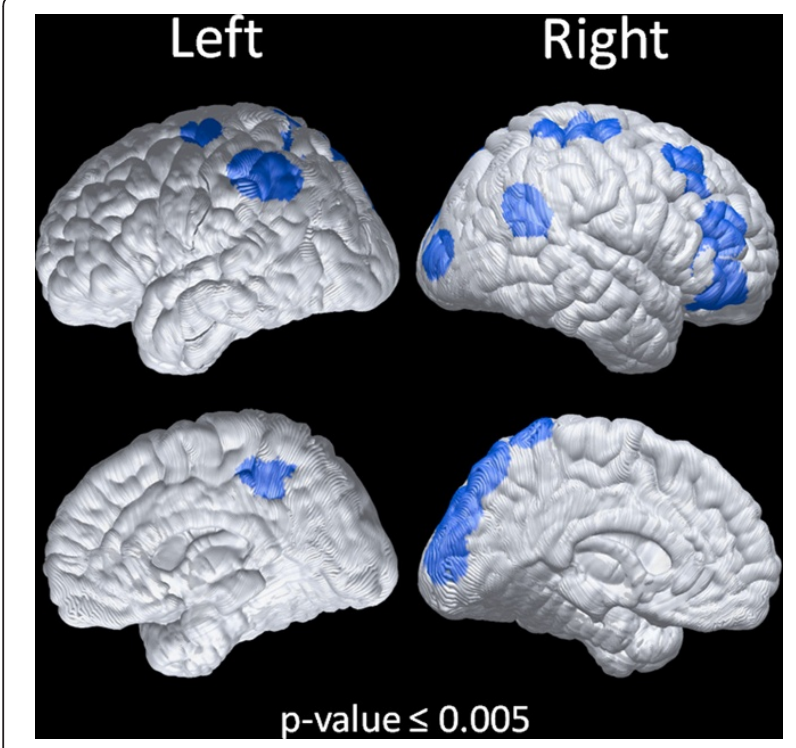

Figure 5 Statistical cortical surface map for voxel-wise group comparison between subjects with and without DLB covarying out the effects of ADAS-Cog. Brain areas of significant

hypometabolism in DLB were shown with an uncorrected threshold of $\mathrm{p} \leq 0.005$.

of DLB and hallucinations, as recorded in the NPI-Q. CSF fluid $A \beta_{1-42}$, $t$-tau and $p$-tau ${ }_{181}$ were associated with the phosphorylated tau (neurofibrillary tangle) Braak stage and the NIA-AA criteria A score, but not with other pathologies. Baseline occipital hypometabolism accurately predicted the presence of DLB pathology, whereas MRI GM occipital atrophy did not. MTL-TDP and AGD were associated with greater hippocampal atrophy.

Despite the neuropathological heterogeneity of the patients, all the demented subjects had a DAT probable clinical diagnosis and the MCI patients had and AD-like profile; three subjects had parkinsonian signs, of those two had DLB pathology and one did not. Therefore, an overall classical DAT presentation does not rule out the presence of coincident vascular disease or other neurodegenerative disease even in the presence of CSF and PET amyloid imaging findings compatible with $\mathrm{AD}$ pathology. This is not surprising because memory impairment is the most common presenting clinical symptom of clinically diagnosed DLB (cDLB) patients (with confirmed abnormal dopamine transporter imaging) [49] and most of the DLB/ $\mathrm{AD}+\mathrm{DLB}$ cases were diagnosed as DAT, showing a low sensitivity of the clinical criteria, at least in this small series [50]. One difference between our study and other previous studies is that we did not include any patients with a CDLB diagnosis and patients with coincident DLB in our study had a typical DAT profile. Nevertheless, we found two clinical markers that were highly predictive of coincident pathologies: a predominant dysexecutive syndrome and the presence of hallucinations. A previous neuropathological study found a high specificity of visual hallucinations in DLB (with a prevalence of $1 \%$ early in the course of $\mathrm{AD}$ ), although prevalence of visual hallucinations was low and therefore not was not a sensitive biomarker [51]. In our study, we found that during the progression of disease a prominence of dysexecutive impairment in the presence of an amnestic profile is a marker for coincident DLB. This is consistent with a previous study that described a worse executive function in subjects with DLB and worse memory in patients with $\mathrm{AD}$, although no classification performance was reported [52]. However, a predominantly disexecutive syndrome might not be a specific biomarker and other NDDs like frontotemporal lobar degeneration could have a similar profile.

Currently, $A \beta$ amyloid PET imaging and CSF $A \beta_{1-42}$ are the most widely accepted research biomarkers for $A D$ which have shown an important correlation with brain $A \beta$ amyloid deposition [20,26,53] and with each other [54]. Confirming the results of our previous study in which one fourth of the patients had coincident pathologies [14], mainly DLB, and studies for other groups [19], CSF $\mathrm{A} \beta$, t-tau and $\mathrm{p}$-tau ${ }_{181}$ levels can reliably predict $\mathrm{AD}$ pathology even in the presence of other coincident pathologies and subjects with $A \beta$ levels above the published cutoff [34] had a low burden of AD.

Three studies with FDG-PET and several neuropathologically confirmed cases have previously reported occipital FDG-PET hypometabolism independent of coincident AD: Albin et al. included three DLB and three with AD + DLB [25], Kantarci et al. included 2 AD and 3 DLB (and a larger number of clinically diagnosed cases) [21] and Minoshima et al. included 7 AD + DLB, 4 DLB and 10 $\mathrm{AD}$ (and a larger number of clinically diagnosed cases) [27]. The last study reported a sensitivity of $90 \%$ and a specificity of $80 \%$ for the diagnosis of DLB with or without AD based on hypometabolism in the occipital cortex [27]. Kantarci et al. carried out a study mostly based on clinically diagnosed patients, and they described an area under the curve (AUC) in the receiver operating characteristic (ROC) of 0.84 for the FDG-PET with cDLB patients showing an occipital hypometabolism independently of $A \beta$ deposition measured by PiB PET [21]. In our study, we found an $80 \%$ sensitivity and a $100 \%$ specificity based on occipital FDG-PET hypometabolism. All of our DLB subjects had coincident $\mathrm{AD}$ and this did not affect the accuracy of the classification. In addition, the only DLB case that was classified as non DLB by the occipital FDG-PET cutoff was the only one that did not have diffuse neocortical LBs. The percentage of predicted DLB pathology in the ADNI-1 DAT subjects was similar to the one observed in the autopsied subjects. Interestingly, it has been suggested that occipital hypometabolism might be an preclinical biomarker of DLB [55]. On the other hand, functional neuroimaging approaches that measure striatal 
dopaminergic innervation and myocardial sympathetic nerve integrity might be more specific to changes associated with LB pathology, specially the latter which captures postganglionic denervation which is present in PD and DLB patients, but not in patients with other atypical parkinsonisms [56]. However, doing these additional tests would increase the, cost, time and inconvenience for patients, whereas FDG PET is also helpful for the differential diagnosis in non-parkinsonian syndromes. Therefore a FDG PET measure that specifically predicts coincident DLB pathology would be preferable. The voxels that contribute to HCI are located in temporal, occipital and parietal cortices and it might be possible that the association with HCI with the presence of DLB and not Braak stage might reflect that this measure is also capturing the posterior cortical metabolism characteristic of DLB. Nevertheless, the AD group without DLB was less impaired at baseline and we did not analyze the FDG PET scans that were matched for clinical severity.

Neither our study nor others have found occipital lobe atrophy in CDLB or DLB $[21,23]$. In addition, we found no differences in hippocampal volume based on the presence of coincident DLB. Conversely, it has been reported that cDLB patients have similar hippocampal volume as $\mathrm{CN}$ subjects but lower hippocampal volume than DAT patients with an high diagnostic accuracy [21] and that a semiquantitative visual rating of MTL MRI atrophy had a high accuracy to classify AD against DLB and pathologically diagnosed vascular cognitive impairment patients [24]. The definition of the DLB group might explain these differences. For example, the study by Burton et al. included cDLB diagnosis patients [24] and the multimodal study by Kantarci et al. was mostly comprised of cDLB patients [21], therefore it can be expected that the pattern of atrophy is different in DLB with a cDLB diagnosis compared to those AD + DLB with a DAT diagnosis. In addition, the study by Kantarci et al. described a large sample of neuropathologically diagnosed subjects in whom only DLB subjects with high DLB probability as defined by McKeith criteria [46] had similar hippocampal volume as $\mathrm{CN}$ subjects, whereas intermediate and low probability DLB had similar hippocampal atrophy as AD subjects [22]. Therefore, hippocampal volume might not be a good marker of coincident DLB in a cohort of DAT subjects.

Late MCI ADNI 1 patients and DAT patients recruited in ADNI represent amnestic, cognitively impaired, subjects without any cognitive signs or symptoms suggestive of non-AD pathologies and a low vascular risk profile [29]. Therefore, these patients with multiple coincident pathologies represent the typical patients recruited in AD clinical trials. These multiple coincident pathologies have important implications for clinical trials and the approach for treating patients. Studies of the brains of patients treated with $A \beta$ immunotherapy have shown a decrease of total $A \beta$ burden and a decrease of neurite curvature ratio and a [57-59] and an increase of amyloid deposition in neocortical blood vessels that might decrease over time [59], without any strong effect on tau [57] or $\alpha$-synuclein [60] clearance. Therefore, multimodal biomarker approaches that aim to detect the different coincident pathologies (instead to categorizing patients into a single diagnostic category), will be needed to select homogenous populations for protein-specific targeted clinical trials and to tailor the treatment for each patient.

\section{Conclusions}

In summary, our study of longitudinally assessed participants in ADNI who had biomarker and postmortem neuropathology found the clinical diagnosis of DAT and $\mathrm{MCI}$ due to $\mathrm{AD}$ was supported by a pathologic diagnosis of $\mathrm{AD}$ in all cases, although some cases had low burden of $\mathrm{AD}$ neuropathologic changes. Although this is a small sample, this observation is consistent with the concept that the clinical spectrum of $\mathrm{AD}$ represents a continuous process. A large proportion of the ADNI AD cases had heterogeneous comorbidities. Whereas CSF AD biomarkers might be able to predict $\mathrm{AD}$ pathology additional biomarkers (neuropsychological profile and FDG-PET) were useful to predict coincident DLB and therefore the combination is useful to predict both pathologies. Hippocampal volume was not a useful biomarker to predict coincident pathologies in this type of patients. These data have implications for the development of biomarkers which are specific for coincident pathologies in addition to $\mathrm{AD}$ and are able to establish the diagnosis of the different pathologies present in the subject. Finally, the presence of frequent comorbidities may help to explain variance in biomarker, structural and functional imaging data and be of utility in explaining altered responses to proposed therapeutic interventions.

\section{Additional file}

Additional file 1: Supplementary Material. Clinical and Multimodal

Biomarker Correlates of ADNI Neuropathological Findings.

\section{Competing interests}

M.W.W.: stock options, Elan, Synarc; travel expenses, Novartis, Tohoku

University, Fundacio Ace, Travel eDreams, MCl Group, NSAS, Danone Trading, ANT Congress, NeuroVigil, CHRU-Hopital Roger Salengro, Siemens,

AstraZeneca, Geneva University Hospitals, Lilly, University of California, San Diego-ADNI, Paris University, Institut Catala de Neurociencies Aplicades, University of New Mexico School of Medicine, Ipsen, Clinical Trials on Alzheimer's Disease, Pfizer, AD PD meeting, Paul Sabatier University; board membership, Lilly, Araclon, Institut Catala de Neurociencies Aplicades, Gulf War Veterans IIInesses Advisory Committee, VACO, Biogen Idec, Pfizer; consultancy, AstraZeneca, Araclon, Medivation = Pfizer, Ipsen, TauRx 
Therapeutics, Bayer Healthcare, Biogen Idec, ExonHit Therapeutics, Servier, Synarc, Pfizer, Janssen; honoraria, NeuroVigil, Insitut Catala de Neurociencies Aplicades, PMDA = Japanese Ministry of Health, Labour, and Welfare, Tohoku University; commercial research support, Merck, Avid; government research support, DOD, VA. Other authors report no conflicts of interest. JM has consulted for Eisai, Glaxo-SmithKline, Novartis, Estavo Jansen Alzheimer Immunotherapy Program, Pfizer and Eli Lilly/Avid Radiopharmaceuticals. D. Aisen serves on a scientific advisory board for NeuroPhage and as a consultant to Elan Corporation, Wyeth, Eisai Inc., Bristol-Myers Squibb, Eli Lilly and Company, NeuroPhage, Merck \& Co., Roche, Amgen, Abbott, Pfizer Inc, Novartis, Bayer, Astellas, Dainippon, Biomarin, Solvay, Otsuka, Daiichi, AstraZeneca, Janssen, Medivation, Inc., Theravance, Cardeus, and Anavex and receives research support from Pfizer Inc., Baxter International Inc.

\section{Authors' contributions}

All authors read and approved the final manuscript, contributed to interpretation of the data and critical review of the manuscript and study concept. NJC and JCM established the neuropathological diagnoses and gradings. DC and EH collected and performed immunohistochemistry. XD and $C D$ processed and analyzed the MRI data. KC, AF, NA, AR, RJB and EMR processed and analyzed the FDG-PET data. JBT drafted the manuscript and performed the statistical analyses. JQT drafted the manuscript.

\section{Acknowledgements}

Data collection and sharing for this project was funded by ADNI (National Institutes of Health Grant U01 AG024904). ADNI is funded by the NIA, NIBIB, and through generous contributions from the following: Alzheimer's Association; Alzheimer's Drug Discovery Foundation; BioClinica, Inc.; Biogen Idec Inc.; Bristol-Myers Squibb Company; Eisai Inc.; Elan Pharmaceuticals, Inc. Eli Lilly and Company; F. Hoffmann-La Roche Ltd and its affiliated company Genentech, Inc.; GE Healthcare; Innogenetics, N.V.; IXICO Ltd.; Janssen Alzheimer Immunotherapy Research \& Development, LLC.; Johnson \& Johnson Pharmaceutical Research \& Development LLC.; Medpace, Inc.; Merck \& Co., Inc.; Meso Scale Diagnostics, LLC.; NeuroRx Research; Novartis Pharmaceuticals Corporation; Pfizer Inc.; Piramal Imaging; Servier; Synarc Inc:; and Takeda Pharmaceutical Company. The Canadian Institutes of Health Research is providing funds to support ADNI clinical sites in Canada. Private sector contributions are facilitated by the Foundation for the National Institutes of Health (www.fnih.org). The grantee organization is the Northern California Institute for Research and Education, and the study is coordinated by the Alzheimer's Disease Cooperative Study at the University of California, San Diego. ADNI data are disseminated by the Laboratory for Neuro Imaging at the University of California, Los Angeles. JQT is the William Maul Measey-Truman G. Schnabel, Jr., Professor of Geriatric Medicine and Gerontology and JCM is Friedman Distinguished Professor of Neurology.

\section{Author details}

${ }^{1}$ Department of Pathology \& Laboratory Medicine, Institute on Aging, Center for Neurodegenerative Disease Research, University of Pennsylvania Perelman School of Medicine, Philadelphia, PA, USA. ${ }^{2}$ Departments of Neurology and Pathology \& Immunology, Washington University School of Medicine, St. Louis, MO, USA. ${ }^{3}$ Section of Biomedical Image Analysis, Department of Radiology, and Center for Biomedical Image Computing and Analytics, University of Pennsylvania, Philadelphia, PA, USA. ${ }^{4}$ Banner Alzheimer's Institute, 901 East Willetta Street, Phoenix, AZ, USA. ${ }^{5}$ University of California at San Diego, San Diego, CA, USA. ${ }^{6}$ Center for Imaging of Neurodegenerative Diseases, Department of Radiology, San Francisco VA Medical Center/ University of California San Francisco, San Francisco, CA, USA.

Received: 23 September 2013 Accepted: 23 September 2013 Published: 9 October 2013

\section{References}

1. Jellinger KA, Attems J: Prevalence of dementia disorders in the oldest-old: an autopsy study. Acta neuropathol 2010, 119:421-433.

2. Bennett DA, Wilson RS, Arvanitakis Z, Boyle PA, de Toledo-Morrell L, Schneider JA: Selected findings from the religious orders study and rush memory and aging project. Journal of Alzheimer's disease: JAD 2013, 33:S397-403.

3. Sonnen JA, Larson EB, Crane PK, Haneuse $S$, Li G, Schellenberg GD, Craft $S$, Leverenz JB, Montine TJ: Pathological correlates of dementia in a longitudinal, population-based sample of aging. Annals of neurology 2007, 62:406-413.

4. Thies W, Bleiler L: 2013 Alzheimer's disease facts and figures. Alzheimers dement j Alzheimers Assoc 2013, 9:208-245.

5. Arnold SE, Toledo JB, Appleby DH, Xie SX, Wang LS, Baek Y, Wolk DA, Lee EB, Miller BL, Lee VM: Trojanowski JQ: A comparative survey of the topographical distribution of signature molecular lesions in major neurodegenerative diseases. J Comp Neurol 2013. Epub 2013/07/25.

6. Mackenzie IR, Neumann M, Bigio EH, Cairns NJ, Alafuzoff I, Kril J, Kovacs GG, Ghetti B, Halliday G, Holm IE, et al: Nomenclature and nosology for neuropathologic subtypes of frontotemporal lobar degeneration: an update. Acta neuropathol 2010, 119:1-4.

7. Montine TJ, Phelps CH, Beach TG, Bigio EH, Cairns NJ, Dickson DW, Duyckaerts C, Frosch MP, Masliah E, Mirra SS, et al: National Institute on Aging-Alzheimer's Association guidelines for the neuropathologic assessment of Alzheimer's disease: a practical approach. Acta neuropathol 2012, 123:1-11.

8. Irwin DJ, White MT, Toledo JB, Xie SX, Robinson JL, Van Deerlin V, Lee VM, Leverenz JB, Montine TJ, Duda JE, et al: Neuropathologic substrates of Parkinson disease dementia. Annals neurol 2012, 72:587-598.

9. Toledo JB, Arnold SE, Raible K, Brettschneider J, Xie SX, Grossman M, Monsell SE, Kukull WA, Trojanowski JQ: Contribution of cerebrovascular disease in autopsy confirmed neurodegenerative disease cases in the National Alzheimer's Coordinating Centre. Brain j neurol 2013, 136:2697-2706.

10. Schneider JA, Boyle PA, Arvanitakis Z, Bienias JL, Bennett DA: Subcortical infarcts, Alzheimer's disease pathology, and memory function in older persons. Annals of neurology 2007, 62:59-66.

11. Chui HC, Zarow C, Mack WJ, Ellis WG, Zheng L, Jagust WJ, Mungas D, Reed BR, Kramer JH, Decarli CC, et al: Cognitive impact of subcortical vascular and Alzheimer's disease pathology. Annals neurol 2006, 60:677-687.

12. Petrovitch H, Ross GW, Steinhorn SC, Abbott RD, Markesbery W, Davis D, Nelson J, Hardman J, Masaki K, Vogt MR, et al: AD lesions and infarcts in demented and non-demented Japanese-American men. Annals neurol 2005, 57:98-103.

13. Beach $T G$, Monsell SE, Phillips LE, Kukull W: Accuracy of the clinical diagnosis of Alzheimer disease at National Institute on Aging Alzheimer Disease Centers, 2005-2010. J neuropathol exp neurol 2012, 71:266-273.

14. Toledo JB, Brettschneider J, Grossman M, Arnold SE, Hu WT, Xie SX, Lee VM, Shaw LM, Trojanowski JQ: CSF biomarkers cutoffs: the importance of coincident neuropathological diseases. Acta neuropathol 2012, 124:23-35.

15. Alladi S, Xuereb J, Bak T, Nestor P, Knibb J, Patterson K, Hodges JR: Focal cortical presentations of Alzheimer's disease. Brain $j$ neurol 2007, 130:2636-2645.

16. James BD, Bennett DA, Boyle PA, Leurgans S, Schneider JA: Dementia from Alzheimer disease and mixed pathologies in the oldest old. JAMA j Am Med Assoc 2012, 307:1798-1800.

17. Schneider JA, Arvanitakis Z, Bang W, Bennett DA: Mixed brain pathologies account for most dementia cases in community-dwelling older persons. Neurology 2007, 69:2197-2204.

18. Jellinger KA, Attems J: Prevalence and impact of vascular and Alzheimer pathologies in Lewy body disease. Acta neuropathol 2008, 115:427-436.

19. Slaets S, Le Bastard N, Theuns J, Sleegers K, Verstraeten A, De Leenheir E, Luyckx J, Martin JJ, Van Broeckhoven C, Engelborghs S: Amyloid pathology influences abeta1-42 cerebrospinal fluid levels in dementia with lewy bodies. J Alzheimers dis JAD 2013, 35:137-146.

20. Tapiola T, Alafuzoff I, Herukka SK, Parkkinen L, Hartikainen P, Soininen $H$, Pirttila T: Cerebrospinal fluid \{beta\}-amyloid 42 and tau proteins as biomarkers of Alzheimer-type pathologic changes in the brain. Archives of neurology 2009, 66:382-389.

21. Kantarci K, Lowe VJ, Boeve BF, Weigand SD, Senjem ML, Przybelski SA, Dickson DW, Parisi JE, Knopman DS, Smith GE, et al: Multimodality imaging characteristics of dementia with Lewy bodies. Neurobiol aging 2012, 33:2091-2105.

22. Kantarci K, Ferman TJ, Boeve BF, Weigand SD, Przybelski S, Vemuri P, Murray ME, Senjem ML, Smith GE, Knopman DS, et al: Focal atrophy on MRI and neuropathologic classification of dementia with Lewy bodies. Neurology 2012, 79:553-560

23. Lebedev AV, Westman E, Beyer MK, Kramberger MG, Aguilar C, Pirtosek Z, Aarsland D: Multivariate classification of patients with Alzheimer's and dementia with Lewy bodies using high-dimensional cortical thickness measurements: an MRI surface-based morphometric study. J neurol 2013, 260:1104-1115. 
24. Burton EJ, Barber R, Mukaetova-Ladinska EB, Robson J, Perry RH, Jaros E, Kalaria RN, O'Brien JT: Medial temporal lobe atrophy on MRI differentiates Alzheimer's disease from dementia with Lewy bodies and vascular cognitive impairment: a prospective study with pathological verification of diagnosis. Brain j neurol 2009, 132:195-203.

25. Albin RL, Minoshima S, D'Amato CJ, Frey KA, Kuhl DA, Sima AA: Fluorodeoxyglucose positron emission tomography in diffuse Lewy body disease. Neurology 1996, 47:462-466.

26. Clark CM, Schneider JA, Bedell BJ, Beach TG, Bilker WB, Mintun MA, Pontecorvo MJ, Hefti F, Carpenter AP, Flitter ML, et al: Use of florbetapirPET for imaging beta-amyloid pathology. JAMA : the journal of the American Medical Association 2011, 305:275-283.

27. Minoshima S, Foster NL, Sima AA, Frey KA, Albin RL, Kuhl DE: Alzheimer's disease versus dementia with Lewy bodies: cerebral metabolic distinction with autopsy confirmation. Annals neurol 2001, 50:358-365.

28. Weiner MW, Veitch DP, Aisen PS, Beckett LA, Cairns NJ, Green RC, Harvey D, Jack CR, Jagust W, Liu E, et al: The Alzheimer's Disease Neuroimaging Initiative: A review of papers published since its inception. Alzheimers dement j Alzheimers Assoc 2013.

29. Petersen RC, Aisen PS, Beckett LA, Donohue MC, Gamst AC, Harvey DJ, Jack CR Jr, Jagust WJ, Shaw LM, Toga AW, et al: Alzheimer's Disease Neuroimaging Initiative (ADNI): clinical characterization. Neurology 2010, 74:201-209.

30. Petersen RC, Smith GE, Waring SC, Ivnik RJ, Tangalos EG, Kokmen E: Mild cognitive impairment: clinical characterization and outcome. Arch neurol 1999, 56:303-308.

31. McKhann G, Drachman D, Folstein M, Katzman R, Price D, Stadlan EM Clinical diagnosis of Alzheimer's disease: report of the NINCDSADRDA Work Group under the auspices of Department of Health and Human Services Task Force on Alzheimer's Disease. Neurology 1984, 34:939-944

32. Gibbons LE, Carle AC, Mackin RS, Harvey D, Mukherjee S, Insel P, Curtis SM, Mungas D, Crane PK: A composite score for executive functioning, validated in Alzheimer's Disease Neuroimaging Initiative (ADNI) participants with baseline mild cognitive impairment. Brain imaging behav 2012, 6:517-527.

33. Crane PK, Carle A, Gibbons LE, Insel P, Mackin RS, Gross A, Jones RN, Mukherjee S, Curtis SM, Harvey D, et al: Development and assessment of a composite score for memory in the Alzheimer's Disease Neuroimaging Initiative (ADNI). Brain imaging behav 2012, 6:502-516.

34. Shaw LM, Vanderstichele H, Knapik-Czajka M, Clark CM, Aisen PS, Petersen RC, Blennow K, Soares H, Simon A, Lewczuk P, et al: Cerebrospinal fluid biomarker signature in Alzheimer's disease neuroimaging initiative subjects. Annals neurol 2009, 65:403-413.

35. Shaw LM, Vanderstichele H, Knapik-Czajka M, Figurski M, Coart E, Blennow K, Soares H, Simon AJ, Lewczuk P, Dean RA, et al: Qualification of the analytical and clinical performance of CSF biomarker analyses in ADNI. Acta neuropathol 2011, 121:597-609.

36. Lindersson E, Beedholm R, Hojrup P, Moos T, Gai W, Hendil KB, Jensen PH: Proteasomal inhibition by alpha-synuclein filaments and oligomers. The Journal of biological chemistry 2004, 279:12924-12934.

37. Fjorback AW, Varming K, Jensen PH: Determination of alpha-synuclein concentration in human plasma using ELISA. Scand j clin lab investig 2007, 67:431-435

38. Jack CR Jr, Bernstein MA, Fox NC, Thompson P, Alexander G, Harvey D, Borowski B, Britson PJ JLW, Ward C, et al: The Alzheimer's Disease Neuroimaging Initiative (ADNI): MRI methods. J magn reson imaging JMRI 2008, 27:685-691.

39. Davatzikos C, Genc A, Xu D, Resnick SM: Voxel-based morphometry using the RAVENS maps: methods and validation using simulated longitudinal atrophy. Neuroimage 2001, 14:1361-1369.

40. Chen K, Ayutyanont N, Langbaum JB, Fleisher AS, Reschke C, Lee W, Liu X, Bandy D, Alexander GE, Thompson PM, et al: Characterizing Alzheimer's disease using a hypometabolic convergence index. Neuroimage 2011, 56:52-60.

41. Cairns NJ, Taylor-Reinwald L, Morris JC: Autopsy consent, brain collection, and standardized neuropathologic assessment of ADNI participants: the essential role of the neuropathology core. Alzheimers dement $j$ Alzheimers Assoc 2010, 6:274-279.

42. The National Institute on Aging, and Reagan Institute Working Group on Diagnostic Criteria for the Neuropathological Assessment of Alzheimer's Disease: Consensus recommendations for the postmortem diagnosis of Alzheimer's disease. Neurobiol aging 1997, 18:S1-2.
43. Beekly DL, Ramos EM, van Belle G, Deitrich W, Clark AD, Jacka ME, Kukull WA: The National Alzheimer's Coordinating Center (NACC) Database: an Alzheimer disease database. Alzheimer dis assoc disord 2004, 18:270-277.

44. Braak H, Alafuzoff I, Arzberger T, Kretzschmar H, Del Tredici K: Staging of Alzheimer disease-associated neurofibrillary pathology using paraffin sections and immunocytochemistry. Acta neuropathol 2006, 112:389-404.

45. Mirra SS: The CERAD neuropathology protocol and consensus recommendations for the postmortem diagnosis of Alzheimer's disease: a commentary. Neurobiol aging 1997, 18:591-94.

46. McKeith IG, Dickson DW, Lowe J, Emre M, O'Brien JT, Feldman H, Cummings J, Duda JE, Lippa C, Perry EK, et al: Diagnosis and management of dementia with Lewy bodies: third report of the DLB Consortium. Neurology 2005, 65:1863-1872.

47. Cairns NJ, Bigio EH, Mackenzie IR, Neumann M, Lee VM, Hatanpaa KJ, White CL 3rd, Schneider JA, Grinberg LT, Halliday G, et al: Neuropathologic diagnostic and nosologic criteria for frontotemporal lobar degeneration: consensus of the Consortium for Frontotemporal Lobar Degeneration. Acta neuropathol 2007, 114:5-22.

48. Hyman BT, Phelps CH, Beach TG, Bigio EH, Cairns NJ, Carrillo MC, Dickson DW, Duyckaerts C, Frosch MP, Masliah E, et al: National Institute on Aging Alzheimer's Association guidelines for the neuropathologic assessment of Alzheimer's disease. Alzheimers dementia j Alzheimers Assoc 2012, 8:1-13.

49. Auning E, Rongve A, Fladby T, Booij J, Hortobagyi T, Siepel FJ, Ballard C, Aarsland D: Early and presenting symptoms of dementia with lewy bodies. Dement geriatr cogn disord 2011, 32:202-208.

50. Nelson PT, Jicha GA, Kryscio RJ, Abner EL, Schmitt FA, Cooper G, Xu LO, Smith CD, Markesbery WR: Low sensitivity in clinical diagnoses of dementia with Lewy bodies. J neurol 2010, 257:359-366.

51. Tiraboschi P, Salmon DP, Hansen LA, Hofstetter RC, Thal LJ, Corey-Bloom J: What best differentiates Lewy body from Alzheimer's disease in earlystage dementia? Brain : a journal of neurology 2006, 129:729-735.

52. Kraybill ML, Larson EB, Tsuang DW, Teri L, MCCormick WC, Bowen JD, Kukull WA, Leverenz JB, Cherrier MM: Cognitive differences in dementia patients with autopsy-verified AD, Lewy body pathology, or both. Neurology 2005, 64:2069-2073.

53. Seppala TT, Nerg O, Koivisto AM, Rummukainen J, Puli L, Zetterberg H, Pyykko OT, Helisalmi S, Alafuzoff I, Hiltunen M, et al: CSF biomarkers for Alzheimer disease correlate with cortical brain biopsy findings. Neurology 2012, 78:1568-1575.

54. Toledo JB, Vanderstichele H, Figurski M, Aisen PS, Petersen RC, Weiner MW, Jack CR Jr, Jagust W, Decarli C, Toga AW, et al: Factors affecting Abeta plasma levels and their utility as biomarkers in ADNI. Acta neuropathol 2011, 122:401-413.

55. Fujishiro H, Iseki E, Kasanuki K, Chiba Y, Ota K, Murayama N, Sato K: A follow up study of non-demented patients with primary visual cortical hypometabolism: Prodromal dementia with Lewy bodies. J Neuro/ Sci 2013. Epub 2013/08/10.

56. Sinha N, Firbank M, O'Brien JT: Biomarkers in dementia with Lewy bodies: a review. Int J Geriatr Psychiatry 2012, 27:443-453.

57. Serrano-Pozo A, William CM, Ferrer I, Uro-Coste E, Delisle MB, Maurage CA Hock C, Nitsch RM, Masliah E, Growdon JH, et al: Beneficial effect of human anti-amyloid-beta active immunization on neurite morphology and tau pathology. Brain j neurol 2010, 133:1312-1327.

58. Holmes C, Boche D, Wilkinson D, Yadegarfar G, Hopkins V, Bayer A, Jones RW, Bullock R, Love S, Neal JW, et al: Long-term effects of Abeta42 immunisation in Alzheimer's disease: follow-up of a randomised, placebo-controlled phase I trial. Lancet 2008, 372:216-223.

59. Boche D, Zotova E, Weller RO, Love S, Neal JW, Pickering RM, Wilkinson D, Holmes C, Nicoll JA: Consequence of Abeta immunization on the vasculature of human Alzheimer's disease brain. Brain j neurol 2008, 131:3299-3310.

60. Bombois S, Maurage CA, Gompel M, Deramecourt V, Mackowiak-Cordoliani MA, Black RS, Lavielle R, Delacourte A, Pasquier F: Absence of beta-amyloid deposits after immunization in Alzheimer disease with Lewy body dementia. Arch neurol 2007, 64:583-587.

doi:10.1186/2051-5960-1-65

Cite this article as: Toledo et al: Clinical and multimodal biomarker correlates of ADNI neuropathological findings. Acta Neuropathologica Communications 2013 1:65. 